\section{POLÍTICAS Y GEOGRAFÍAS DEL DESPLAZAMIENTO. CONTEXTOS Y USOS CONCEPTUALES PARA EL DEBATE SOBRE GENTRIFICACIÓN*}

Jorge Blanco**, Ricardo Apaolaza***

\section{Resumen}

El trabajo se propone realizar una reflexión sobre los usos, contenidos, articulaciones e implicancias políticas del concepto de desplazamiento, en el marco de la geografía humana, con la finalidad última de ubicar el debate sobre gentrificación dentro del complejo mapa del desplazamiento a escala global. Persiguiendo una finalidad analítica, y sobre la base de los resultados empíricos de una amplia revisión bibliográfica, el trabajo organiza los usos del término desplazamiento en siete grandes constelaciones temáticas: 1) asociado a la movilidad urbana y el transporte; 2) asociado a la movilidad residencial; 3) asociado a las migraciones; 4) asociado a fenómenos naturales; 5) asociado a

\section{POLICIES AND GEOGRAPHIES OF DISPLACEMENT. CONTEXTS AND CONCEPTUAL USES FOR THE GENTRIFICATION DEBATE*}

Jorge Blanco**, Ricardo Apaolaza***

\section{Abstract}

This paper reflects on the use, contents, relationships and political consequences of the concept of displacement within the context of human geography in order to place the debate on gentrification in the complex map of displacement at a global scale. By following an analytical approach and based on the empiric findings of a bibliographic review, the present paper organizes the use of the displacement concept into seven major thematic groups: 1) urban mobility and transport; 2) residential mobility; 3) migration; 4) extreme natural events; 5) political-military conflicts and tensions; 6) infrastructure and territorial planning projects; and 7) gentrification and urban 
conflictos y tensiones político-militares; 6) asociado a proyectos de infraestructura y ordenamiento territorial; y 7) asociado a los procesos de gentrificación y renovación urbana. Se concluye que la diversidad de situaciones en las que el término desplazamiento es utilizado refleja la centralidad que los movimientos de población adquieren en el análisis de los actuales procesos socio-territoriales, y que los estudios sobre gentrificación pueden enriquecerse con los resultados de otros estudios sobre desplazamiento, ya que todos ellos pueden ser entendidos como parte de un debate mayor asociado a la desposesión a escala planetaria.

\section{PALABRAS CLAVE: DESPLAZAMIENTO; GENTRIFICACIÓN; DESPOSESIÓN; GEOGRAFÍA}

Recibido: 08-03-2016

Aceptado: 30-09-2016

* Trabajo desarrollado en el marco del proyecto “CONTESTED CITIES. Contested Spatialities of Urban Neoliberalism", financiado por la Comisión Europea (Grant Agreement PIRSES-GA-2012-318944).

* Argentina. Profesor en geografía. Magister en políticas ambientales y territoriales (Universidad de Buenos Aires). Docente e investigador del Instituto de Geografía de la Universidad de Buenos Aires. Correo electrónico: jblanco@filo.uba.ar.

** Argentina. Licenciado y profesor en geografía (Universidad de Buenos Aires). Becario doctoral de la Universidad de Buenos Aires. Correo electrónico: ricardo.apaolaza@filo.uba.ar. renewal processes. This study concludes that the diversified uses of the displacement concept show the importance of population movement within the analysis of current socio-territorial processes. It is also concluded that research on gentrification may benefit from the findings of other studies on displacement since they refer to a larger debate associated with dispossession on a global scale.

\section{KEYWORDS: DISPLACEMENT; GENTRIFICATION; DISPOSSESSION; GEOGRAPHY}

Received: 08-03-2016

Accepted: 30-09-2016

* This research is part of the project entitled "CONTESTED_CITIES. Contested Spatialities of Urban Neoliberalism", funded by the European Commission (Grant Agreement PIRSES_GA-2012-318944).

* Argentina. Professor of Geography. MSc in Environmental and Territorial Policies (University of Buenos Aires). Professor and researcher, Institute of Geography, University of Buenos Aires. Email: jblanco@filo.uba.ar

*** Argentina. BA and professor of Geography (University of Buenos Aires). Doctoral fellow at University of Buenos Aires. Email: ricardo.apaolaza@filo.uba.ar. 


\section{Introducción}

El trabajo se propone realizar una reflexión sobre los usos, contenidos, articulaciones e implicancias políticas del concepto de desplazamiento, en el marco de la geografía humana, con la finalidad última de aportar nuevas herramientas e ideas al debate de la gentrificación.

El amplio desarrollo reciente de los estudios sobre gentrificación ha puesto en el centro de la discusión la cuestión de los desplazamientos, tanto como contenido analítico como por ser objeto de disputas políticas. Se trata de un término muy utilizado, que por su amplitud y versatilidad es pasible de diversas interpretaciones y usos. ¿En qué contextos se lo utiliza? ¿Qué usos adopta el término en cada uno de esos contextos? ¿Cómo se vinculan esos usos con los existentes en los estudios sobre gentrificación? ¿Cómo se puede religar la variedad de sentidos dispersos del término desplazamiento en el contexto de los actuales procesos socio-territoriales? Para intentar responder estas preguntas se propone una tarea de revisión, análisis y ordenamiento de bibliografía existente sobre el tema.

Los resultados del análisis de los trabajos revisados a partir del sentido -explícito o implícitootorgado al término desplazamiento permitieron identificar siete grandes constelaciones ${ }^{1}$ : 1) Desplazamiento asociado a la movilidad urbana y el transporte; 2) Desplazamiento asociado a la movilidad residencial; 3) Desplazamiento asociado a las migraciones; 4) Desplazamiento asociado a fenómenos naturales; 5) Desplazamiento asociado a conflictos y tensiones político-militares; 6) Desplazamiento asociado a proyectos e intervenciones de infraestructura y ordenamiento territorial; y 7) Desplazamiento asociado a procesos de gentrificación y renovación urbana.

A partir de estos grandes agrupamientos, que se presentan y discuten en los apartados siguientes, se intenta enriquecer el debate sobre desplazamiento y gentrificación.

\section{Consideraciones metodológicas}

El trabajo partió de una amplia revisión de literatura académica en español, portugués, francés e inglés, para posteriormente identificar y analizar algunos grandes agrupamientos recurrentes basados en el uso efectivo del término desplazamiento, fundamentalmente en sus dimensiones residenciales.

\footnotetext{
Constelación, entendida como un agrupamiento sobre un núcleo de uso específico, en torno al cual gravitan también otros trabajos emparentados o con usos cercanos.
} 
De esta manera, la estrategia metodológica se basó en una doble revisión bibliográfica -focalizada en 27 revistas especializadas y a través de 8 motores de búsqueda-, con la que se encontraron más de 500 trabajos que hacían un uso intensivo o estratégico del término "desplazamiento", y que fueron comunicados dentro de ámbitos y publicaciones pertenecientes al campo de la geografía.

Tal cual observa Delgadillo ${ }^{2}$, se pueden identificar al menos una veintena de sinónimos de variados sentidos y connotaciones. A pesar de esto, la revisión empírica de los trabajos puso en evidencia que, de una u otra manera, desplazamiento está asociado a dos grandes ideas: movimiento o expulsión. Aunque a simple vista parecería tratarse de dos acepciones independientes, los límites entre uno y otro sentido no son rígidos ni, mucho menos, exactos. Se trata de dos usos que, en rigor, remiten a los dos sentidos contenidos en la genética etimológica del término3:

- Desplazar, como idea de cambio de plaza o lugar.

- Desplazar, como idea de pérdidalcarencia de plaza o lugar.

Mientras que en el primer sentido predomina una idea de traslado, movimiento o viaje a través del

2 Delgadillo, 2015.

3 Según la RAE, de des- (prefijo de pérdida/carencia o bien de externalización), y plaza (como "sitio determinado para una persona o cosa" o, en un sentido más general, "espacio, sitio o lugar"). espacio geográfico, en el segundo sentido predomina una idea de posibilidad de estancia o permanencia en el espacio geográfico. Asimismo, mientras que el primer sentido habilita la posibilidad de un modo activo y uno pasivo (es posible trasladarse tanto como ser trasladado), el segundo parecería favorecer únicamente un modo pasivo (sólo es posible perder la plaza a manos de alguien o algo). Es importante señalar que la equivalencia en inglés del término (displacement) sólo da cuenta del segundo sentido, es decir que siempre connota algún grado de coerción.

Como se verá, estos sentidos de una u otra manera subyacen y reaparecen -con diferentes connotaciones e implicancias- en las grandes perspectivas y líneas temáticas identificadas en la revisión.

\section{Resultados: contextos y usos del concepto de desplazamiento para el debate de la gentrificación}

Las siete constelaciones identificadas por el presente trabajo presentan estrechas vinculaciones entre sí y habitualmente aparecen reunidas bajo un 
enfoque común. Además, muchos de estos agrupamientos en gran medida coinciden con aquellas temáticas discutidas en las agendas de organismos internacionales como el Banco Mundial o el Banco Interamericano de Desarrollo ${ }^{4}$.

Una rápida idea de las principales vinculaciones puede observarse en la figura 1.

Se realiza a continuación una breve presentación de cada uno de estos agrupamientos, destacando sus principales elementos constitutivos y atributos, así como sus potenciales aportes para pensar el fenómeno de la gentrificación.

\section{DESPLAZAMIENTO ASOCIADO A LA MOVILIDAD URBANA Y EL TRANSPORTE}

La primera constelación se organiza en torno al campo de la movilidad urbana y el transporte, donde el uso del término desplazamiento remite, primeramente, al movimiento físico, es decir una simple unidad de medida de la movilidad.

Aquí la movilidad cotidiana es entendida como el conjunto de los desplazamientos recurrentes realizados en la escala de la cotidianeidad, y es concebida como una necesidad y como un derecho ${ }^{5}$, ya que da cuenta de las posibilidades de acceso a los servicios básicos para el desarrollo de la vida

\section{Terminski, 2013.}

5 Ascher, 2004. urbana, la participación activa en los procesos económicos y la socialización de las personas.

La movilidad pone en juego las necesidades de los sujetos para desarrollar sus actividades de reproducción de la vida social, en un cierto contexto territorial y temporal ${ }^{6}$. Gutiérrez la define como "una práctica social del viaje que conjuga deseos y necesidades de desplazamiento (que en conjunto pueden definirse como requerimientos de movilidad) y capacidades de satisfacerlos"7, y de su interacción resultan las condiciones de accesibilidad de distintos grupos sociales. Esta definición enfatiza que se trata de una práctica en el territorio con una frecuencia de realización, que define un patrón de desplazamiento en un contexto social, espacial y temporalmente determinado.

En términos de las escalas espaciales de los movimientos cotidianos, se estudian los desplazamientos de proximidad y de alcance urbano, metropolitano o regional, según los casos. Se ponen en juego así distintos procesos de apropiación y uso de la ciudad y la posibilidad de acceso a los recursos metropolitanos ${ }^{8}$.

Como se desprende de estas rápidas observaciones, estos trabajos utilizan el término desplazamiento de manera instrumental, para dar cuenta de la acción física del movimiento. En los estudios clásicos

\footnotetext{
$6 \quad$ Levy, 2000.

$7 \quad$ Gutiérrez, 2012, p. 67.

8 Blanco, Bosoer y Apaolaza, 2014.
} 


\section{FIGURA 1. CONSTELACIONES EN TORNO AL USO DEL CONCEPTO DE DESPLAZAMIENTO}

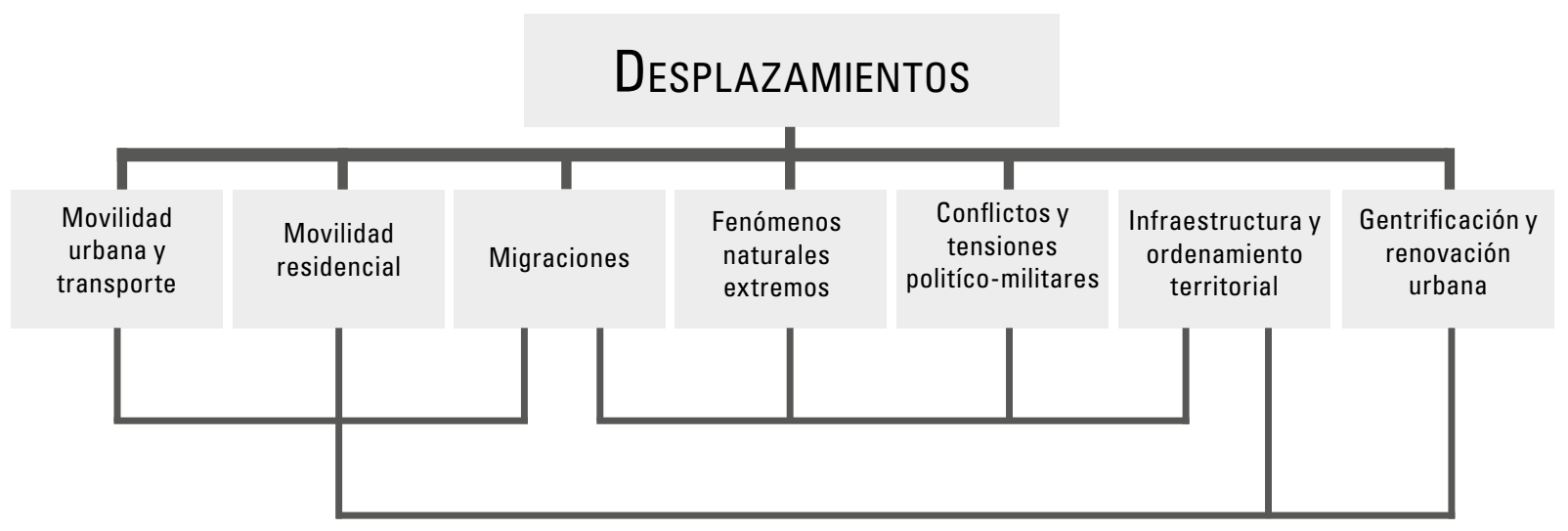

Fuente: elaboración propia.

de transporte, los desplazamientos constituyen apenas una unidad de medida, más o menos equivalente al viaje. Es en cambio el término movilidad el que articula novedosas perspectivas de investigación, ya que aborda esos desplazamientos como prácticas sociales en el espacio, abriendo nuevas posibilidades: las actuales perspectivas sitúan los desplazamientos urbanos en espacios relacionales que constituyen la trama en la que se desarrolla la vida cotidiana, vinculando así fijación y movimiento, de modo que cambios en la residencia implican cambios en las movilidades cotidianas.
Esta constelación aporta elementos para analizar los efectos del desplazamiento por gentrificación sobre las posibilidades de uso y apropiación de la ciudad. Los desplazados ven afectados todo el sistema de lugares articulados por la movilidad cotidiana $^{9}$, con una doble consecuencia. Por un lado, muchos de ellos están obligados a realizar viajes que insumen más tiempo y a un costo más elevado para atender a las necesidades de movilidad. Por otro lado, algunos de los grupos desplazados quedan limitados a una movilidad de proximidad, con efectos negativos sobre las posibilidades de satisfacer sus necesidades cotidianas, o accediendo

9 Dureau, 2002 
a empleos informales en las cercanías de la nueva residencia ${ }^{10}$.

\section{DESPLAZAMIENTO ASOCIADO A LA MOVILIDAD RESIDENCIAL}

La segunda constelación se estructura sobre una línea tradicional de la demografía y la sociología que estudia la movilidad residencial, entendida como "aquellas prácticas espaciales que involucran cambios en el lugar de residencia en la ciudad"11, y entiende el desplazamiento fundamentalmente como un cambio de localización sin atribuir, al menos de antemano, un sentido compulsorio o expulsivo.

Las aproximaciones tradicionales de esta línea han puesto un fuerte énfasis cuantitativo en dimensionar los movimientos que se producen en la movilidad residencial o en asociarlos con la dinámica del mercado inmobiliario o del ciclo de vida de las familias, en tanto que posteriores aproximaciones insertaron esos cambios de emplazamiento en entramados socioterritoriales más complejos, como los derivados de las posibilidades de elección residencial que tienen los distintos grupos sociales ${ }^{12}$.

\footnotetext{
10 Suárez, Murata y Delgado, 2016.

11 Di Virgilio, 2014, p. 13.

12 Dureau, 2002.
}

En perspectivas más recientes, se destaca que la movilidad residencial está asociada en sistemas de movilidad de distintas escalas temporales y espaciales y, en esa línea, se vincula con las migraciones y/o con la movilidad cotidiana ${ }^{13}$.

La localización residencial es también vista como parte de los recursos de las familias, "en la medida que hace variar las ventajas ofertadas por el entorno de proximidad para facilitar la organización de la vida cotidiana"14. Los lugares de vida que ofrecen pocos servicios obligan a la dispersión espacial de las actividades del hogar, lo que puede conllevar costos organizacionales tan altos como para justificar una mudanza.

Módenes propone dos ideas innovadoras para pensar la movilidad residencial: a) relacionar los habitantes con la totalidad del territorio que utilizan; y b) relacionar los lugares con la totalidad de sus usuarios. Para relacionar a los habitantes con la totalidad del territorio que utilizan, es de relevancia el concepto de sistema residencial, entendido como "la coordinación de las estrategias espaciales de los miembros de las redes familiares extensas ya sea por el mantenimiento de un cierto nivel de proximidad residencial (...) o por la coordinación de] movilidades residenciales y habituales de distintos miembros"15. Con similares características

\footnotetext{
13 Módenes, 2008.

14 Thomas, Pattaroni y Kaufmann, 2011, p. 4-5.

15 Dureau, 2002
} 
se utiliza el concepto de espacios de vida, que se define como "todos aquellos lugares organizados alrededor de la residencia y frecuentados habitualmente por el individuo"16. Por su parte, para relacionar a los lugares con la totalidad de los usuarios, es de relevancia la idea de apropiación y uso del territorio, que da cuenta de un conjunto amplio de desplazamientos por transformaciones en el mercado laboral, en las condiciones de oferta comercial y de consumo o por distintos mecanismos de restricción de acceso ${ }^{17}$.

Como síntesis, los trabajos sobre movilidad residencial asocian diferentes formas y escalas de la movilidad, tradicionalmente vistas como separadas (migraciones, movilidad cotidiana, etc.). Si bien en esos trabajos también predomina un uso auxiliar del término desplazamiento, que aparece como acción física sin más connotaciones que la del mero movimiento, algunas discusiones dentro de esta línea adquieren relevancia para el debate de la gentrificación: la relación entre fijación y movilidad, la dimensión territorial y temporal de esos desplazamientos, las condiciones y posibilidades de decidir la localización residencial, y la relación de la movilidad residencial con la diferenciación social. Esta constelación también arroja valiosas pistas sobre cómo pueden repercutir los

$16 \quad$ Courgeau 1988 en Módenes, 2008.

17 Veschambres, 2005; Blanco, Bosoer y Apaolaza, 2014. desplazamientos por gentrificación no sólo sobre los lazos sociales, sino también sobre las posibilidades de uso y apropiación de la ciudad.

\section{DESPLAZAMIENTO ASOCIADO A LAS MIGRACIONES}

Una tercera constelación en el uso del término desplazamiento se organiza en torno al estudio de las migraciones. Dentro de este subgrupo, un porcentaje mayoritario de trabajos utiliza el vocablo desplazamiento cuando requiere un término auxiliar al de migración para dar cuenta del movimiento de personas en un sentido general o "neutro"18, y en ocasiones se termina por equiparar desplazamiento a movimiento. Se trata de usos espontáneos en los cuales el término no reviste un carácter estrictamente conceptual.

Diversos aspectos aparecen analizados en la bibliografía específica sobre desplazamientos en el marco de las migraciones. Por un lado la idea de que ese desplazamiento es "significativo", vinculando a las personas con los lugares que habitan y con las trayectorias pasadas y futuras del movimiento ${ }^{19}$. Por otro lado, se aborda la territorialidad de los migrantes, atendiendo a las fijaciones y movilidades que implican cambios en los contextos en que se insertan ${ }^{20}$. Se propone, además, una discusión

18 Abellán y Rojo, 1997; Brito, 2006.

19 Veleda, 2001.

20 Brachet, 2008. 
relevante sobre la escala temporal (permanencia, recurrencia, etc.) y espacial (rural-urbana, nacional, internacional) del desplazamiento migratorio.

Sin embargo, el término desplazamiento también aparece con un segundo sentido para dar cuenta de migraciones de carácter forzoso. Varios trabajos proponen diferenciar entre migraciones voluntarias, cuando es el migrante quien ha decidido, y forzadas, si el desplazamiento le ha sido impuesto $^{21}$. Coincidentemente Ruiz entiende que la característica distintiva del desplazamiento tiene que ver con las fuerzas de expulsión y no con las de atracción. De esta manera, el carácter forzado le da una connotación particular frente a otros tipos de migraciones, ya que "es una migración no voluntaria, no determinada por la oferta de mejores condiciones laborales o sociales, [...] forzada por las condiciones que se desarrollan en el territorio donde se habita"22.

Sin embargo, los límites entre uno y otro escenario no son claros, las causas no aparecen en estado puro y la distinción entre los factores coercitivos se torna difícil ${ }^{23}$. De esta manera, por ejemplo la desarticulación de los circuitos productivos generada como efecto colateral de proyectos de desarrollo o conflictos armados, puede ser fundamental en tanto que factor expulsivo, aun sin que haya un hostigamiento directo sobre la población.

En síntesis, dentro de este subgrupo coexisten elementos de los dos sentidos etimológicos del desplazamiento (como cambio y pérdida de plaza). Mientras un porcentaje mayoritario de trabajos lo utiliza de manera espontánea cuando necesita un término auxiliar al de migración para dar cuenta del movimiento, otro grupo lo asocia con la coerción y lo compulsivo. Una importante idea que se extrae, es la de la inexistencia de unos supuestos límites precisos entre una relocalización voluntaria asociada a factores económicos de atracción, y otra forzada asociada a factores políticos de expulsión, destacando el papel de la presión económica, la destrucción del tejido social y productivo o la existencia de redes de apoyo en la decisión de resistir o emigrar. Procesos y rasgos similares pueden ser analizados en relación con los desplazamientos originados en procesos de gentrificación.

\section{DESPLAZAMIENTO ASOCIADO A FENÓMENOS NATURALES}

Este agrupamiento entiende el desplazamiento como un cambio forzado de residencia, lugar de vida y/o lugar de trabajo, algo que, como se verá,

21 Echavarría, 1980, Silvey, 2004.

22 Ruiz, 2008, p. 5.

23 Osorio, 2001, p. 13. 
comparte con las siguientes constelaciones. La particularidad de este subgrupo radica en su interés por aquellos móviles causales vinculados a lo que podría denominarse genéricamente "catástrofes sociales desencadenadas por fenómenos naturales". El interés está tanto en los desplazamientos pasados y presentes asociados a eventos extremos, como en aquellos esperados a futuro, con el cambio climático como telón de fondo y los pronósticos de más frecuentes e intensos eventos catastróficos. Emparentados con la noción central dentro de esta línea -el "desplazamiento ambiental"-, aparecen otras expresiones o neologismos como "desplazado climático"24, "migración ambiental"25, "refugiado climático"26 o "climigración"27.

Se detectan también algunas distinciones interesantes que abordan la cuestión temporal no desde la estacionalidad o la durabilidad, sino desde la reversibilidad o posibilidad de retorno al lugar de origen: desplazamiento de "corto término", de "mediano término" o "permanente"28. Vinculado a estas distinciones, aparece también la tensión forzado-voluntario, mencionándose desplazamientos "precautorios" (auto-evacuaciones frente a la advertencia de un evento extremo), y "de emergencia” (efectuados como reacción al evento).

\footnotetext{
24 Barua y Shahjahan, 2013.

25 Oliver-Smith, 2012.

26 Myers, 1997.

27 Bronen, 2010

28 Black, Arnell, Adger, Thomas, y Geddes, 2013.
}

Asimismo, al igual que en los estudios sobre migraciones, Kolmannskog y Trebbi discuten sobre los difusos límites entre lo estrictamente voluntario y lo compulsivo: "¿Qué nivel de degradación debe alcanzar la tierra para considerar que los pequeños agricultores se han visto forzados a abandonarla y no que meramente 'han optado' por dejarla en pos de una vida mejor en otro lugar?"29. Terminski distingue entre un desplazamiento causado por "desastres naturales repentinos" de otro causado por "cambios ambientales de evolución lenta", planteando que en el primer caso las personas tienden a retornar una vez que la situación crítica ha pasado, mientras que en el segundo suele darse un abandono definitivo ${ }^{30}$.

Otro punto importante tiene que ver con el "factor activo" del desplazamiento, esto es el fenómeno o proceso al cual se le atribuye la motorización del desplazamiento: en algunos trabajos se plantea que es el evento extremo, el "cambio climático", etc., quien produce el desplazamiento ${ }^{31}$, mientras que en otros se incluyen condicionantes sociales y político-económicos ${ }^{32}$, o hasta se analiza cómo diferentes actores dominantes hacen uso de la "oportunidad" abierta por el episodio catastrófico para expandir sus posibilidades de negocios, para

29 Kolmannskog y Trebbi, 2010, p. 310.

30 Terminski, 2013, p. 12.

31 Kolmannskog y Trebbi, 2010.

32 Ruwanpura, 2009. 
"reordenar" la estructura territorial o simplemente para reforzar su control territorial ${ }^{33}$. En esta última línea, algunos autores abordan el desplazamiento ambiental desde la idea de disaster capitalism trabajada por Naomi Klein ${ }^{34}$. Esta lógica oportunista vinculada a los "desastres naturales" también ha sido identificada para procesos de gentrificación ${ }^{35}$.

Finalmente, Ruwanpura destaca la importancia de las estructuras sociales cristalizadas al momento de diagramar programas de reasentamiento por parte del Estado ${ }^{36}$, algo que coincide con los tradicionales manuales y modelos sobre desplazamiento y reasentamiento ${ }^{37}$.

Se rescatan de este agrupamiento algunas ideas que despiertan interesantes interrogantes para los debates de la gentrificación: nuevamente la tensión voluntario-forzado, el interés por prevenir futuros desplazamientos antes que por describir aquellos ya en curso, la posibilidad de revertir el proceso, el anonimato causal que oculta responsabilidades políticas o económicas y la utilización del detonante físico-natural para la apertura de procesos de mercado, como ejemplifican los desarrollos inmobiliarios y turísticos en diferentes ciudades.

33 Hyndman, 2007.

34 Timms, 2011.

35 Inzulza y López, 2014.

36 Ruwanpura, 2009.

37 Scudder y Colson, 1982; Cernea, 1997.

\section{DESPLAZAMIENTO ASOCIADO A CONFLICTOS POLÍTICO-MILITARES}

Los trabajos que abordan el desplazamiento forzado relacionado con conflictos militares o represión política se caracterizan por cubrir una gran diversidad de casos de todo el mundo. Entre ellos, se destaca el caso colombiano, donde en un contexto de desplazamiento masivo ha habido un creciente reconocimiento por parte del Estado, a través de la incorporación de la figura del desplazado dentro de la legislación nacional ${ }^{38}$ o de la creación de un registro oficial de desplazados ${ }^{39}$.

Dentro de esta constelación, interesan fundamentalmente cuatro aportes. Primero, queda explícitamente evidenciada la responsabilidad política así como el carácter injusto e ilegal del desplazamiento, algo que aún es discutido en los casos asociados a desastres naturales o mega-proyectos. Muggah entiende que los desplazamientos inducidos por conflictos suelen ser "espontáneos, imprevisibles e ilegales para el derecho internacional”, a la vez que el reasentamiento es descoordinado y suele ser visto por los responsables políticos como una situación temporal, aún cuando termina por ser permanente ${ }^{40}$.

\footnotetext{
38 Ver Ley 387/97 sobre prevención del desplazamiento forzado.

39 Registro Único de Personas Desplazadas (RUPD).

40 Muggah, 2003, p. 15.
} 
Segundo, hay un abordaje más detallado de la tensión "forzado-voluntario". Victoria y Molina diferencian desplazamientos forzosos - preventivos ante el riesgo-, de aquellos forzados -posteriores a la ocurrencia de las acciones ${ }^{41}$. Egea y Soledad, recuperan aportes en torno a esta tensión que distinguen migraciones libres, obligadas, forzadas, forzosas, espontáneas y dirigidas; destacando que el desplazamiento no sólo es involuntario sino que además, conlleva el desconocimiento del lugar de llegada ${ }^{42}$. Penz también plantea que "si es voluntario, entonces no es desplazamiento", ya que la elección de quedarse es central en la dicotomía ${ }^{43}$. No obstante, justamente por tratarse de una elección, es por definición subjetiva, por lo que algo que puede ser presentado como voluntario desde lo formal, puede ser involuntario en la práctica ${ }^{44}$.

Tercero, se resalta la dificultad para analizar aisladamente las causas que generan el desplazamiento ${ }^{45}$. Hay cierto consenso en que dentro de los contextos de conflicto político-militar, la violencia directa no es la única causa del desplazamiento, sino que se conjuga con factores económicos (destrucción de las condiciones de producción, desarticulación de las redes de comercialización, etc.) o perceptivos (sensación de inseguridad, falta de

\footnotetext{
41 Victoria y Molina, 2003.

42 Egea y Soledad, 2008.

43 Penz, 2003, p. 3.

44 Muggah, 2003.

45 Ruiz, 2008.
}

expectativas, etc.), que frecuentemente dependen de grandes agentes económicos asociados a megadesarrollos extractivos.

Por último, se estudian con mucho detalle los efectos del desplazamiento sobre los grupos desplazados. Brun analiza cómo muchos desplazados o refugiados pueden estar físicamente presentes en un lugar, pero a la vez ser parte de comunidades trans-locales ${ }^{46}$. Osorio identifica temporalidades inciertas entre el "ser" y "estar" desplazado, donde el desplazamiento "no se asume como una situación transitoria, sino que se instaura como identidad esencial ${ }^{47}$.

Dentro de esta constelación se abarca una gran diversidad geográfica de casos y situaciones, y se evidencia la importancia de resaltar la responsabilidad política puesta en juego en el desplazamiento, así como las posibilidades de resistencia en torno a la construcción jurídica de la figura del desplazado. Además, estos trabajos aportan un refinamiento de la discusión de la voluntariedad y arrojan pistas sobre cuestiones identitarias en la condición de desplazado. Estas cuestiones no son habitualmente abordadas por los estudios sobre desplazamiento por gentrificación.

$46 \quad$ Brun, 2001.

47 Osorio, 2001. 


\section{DESPLAZAMIENTO ASOCIADO A PROYECTOS DE INFRAESTRUCTURA Y ORDENAMIENTO TERRITORIAL}

Una sexta constelación se organiza en torno al análisis del desplazamiento asociado a proyectos e intervenciones de ordenamiento territorial e infraestructura. Se trata de trabajos que entienden el desplazamiento como un cambio involuntario de lugar de vida, pero que abarcan situaciones en las cuales el propio Estado, de manera directa o indirecta (a través de permisos, concesiones, legislación, etc.), desencadena o comanda el desplazamiento. Así, el factor activo del desplazamiento posee necesariamente un componente políticoeconómico centralizado y explícito.

En este agrupamiento, el término desplazamiento suele aparecer conjuntamente con el de "reasentamiento", esto es, la relocalización permanente más o menos forzada de población dentro del mismo territorio nacional a manos de la autoridad estatal. Numerosos autores refieren a este tipo de proceso como "desplazamiento y reasentamiento inducido por el desarrollo" o "DIDR", y lo interpretan como una situación habitual desde la década de 1950 en grandes proyectos de represas, minería,

48

Development-induced displacement and resettlement, en inglés. infraestructura urbana y emprendimientos turísticos, entre otros ${ }^{49}$.

A su vez, se suele afirmar que dado que la mayoría de estas intervenciones se financian con créditos de organismos internacionales (Banco Mundial, Banco Interamericano de Desarrollo, etc.), los escenarios de desplazamiento serían más suaves o hasta auspiciosos, ya que estos organismos "incluyen dentro de los contratos de empréstito, aspectos relacionados con el objetivo del reasentamiento y la metodología a implementar pretendiéndose un mejoramiento de la calidad de vida de la población" ${ }^{50}$. Sin embargo, otros trabajos entienden que el accionar del Estado o del sector privado trasnacional, aún bajo las salvaguardas de los organismos crediticios, no necesariamente conlleva una contemplación, ni menos aún una mejora, de las condiciones de vida de la población desplaza$\mathrm{da}{ }^{51}$. Vainer, propone incluso la expresión "guerra del desarrollo" para referir a ese tipo de desplazamientos, afirmando que las acciones llevadas a cabo en nombre del progreso han sido "tan implacables como las guerras propiamente dichas" 52 .

Fenner Sánchez, denuncia que las reubicaciones por proyectos de desarrollo son muchas veces utilizadas como "herramienta del ordenamiento

\footnotetext{
49 Muggah, 2003; Terminski, 2013.

50 Victoria y Molina, 2003, p. 20.

51 Zalik, 2009; Fenner Sánchez, 2011.

52 Vainer, 1998, p. 821.
} 
territorial" 53 , algo que en parte se comprueba en procesos de relocalización de slums ${ }^{54}$. También se cuestionan los límites entre las relocalizaciones voluntarias y las involuntarias, ya que "la mayoría de las veces la población es conminada a desplazarse, aunque en ocasiones, dado el proceso de negociación y convencimiento que se sigue, estos casos podrían presentarse como desplazamientos voluntarios" $" 55$.

Frente a este escenario, la discusión sobre estos efectos negativos ha tendido a generar dos polos: por un lado quienes consideran que el desplazamiento es un "efecto inevitable, aunque quizá no intencionado, del desarrollo"; y por otro quienes consideran que el desplazamiento es un "inaceptable fracaso del mismo"56.

En síntesis, estos trabajos destacan un aspecto no tan explícito en los estudios de desplazamiento por gentrificación: la plena responsabilidad del Estado, a la vez que su deber (al menos formal) para con el reasentamiento de los desplazados. También alertan sobre las políticas de "ingeniería social" que pueden subyacer los procesos de desplazamiento y reasentamiento comandados por el Estado, fundamentales también en la gentrificación "stateled", pero a la vez muestran las posibilidades que

Fenner Sánchez, 2011, p. 60.

Kamete, 2012; Johnston, 2014.

Fenner Sánchez, 2011, p. 62.

Muggah, 2003, p. 11-12. podrían derivarse del reconocimiento (legal, prescriptivo) de la responsabilidad estatal o del reasentamiento obligatorio. Las acciones vinculadas con los proyectos de infraestructura y ordenamiento territorial han originado importantes movimientos de resistencia ciudadana protagonizados por las poblaciones desplazadas o por diferentes organizaciones de la sociedad civil ${ }^{57}$.

\section{DESPLAZAMIENTO ASOCIADO A LOS PROCESOS DE GENTRIFICACIÓN Y RENOVACIÓN URBANA}

Esta última constelación representa el núcleo central de interés del presente artículo, ya que agrupa los trabajos que utilizan el término desplazamiento para dar cuenta de procesos de gentrificación y expulsión en el ámbito urbano.

El desplazamiento suele ser parte integral de la definición de gentrificación ${ }^{58}$ y representa una dimensión clave en la discusión crítica. Janoschka y otros han destacado el sentido político que el desplazamiento otorga al debate de la gentrificación, proponiendo el uso conjunto de ambos términos como una manera de destacar la perspectiva política de la investigación científica ${ }^{59}$. Slater propuso recuperar el debate del desplazamiento como

57 Gómez, Wagner, Torres, Martin, y Rojas, 2014; Flores, 2015

58 Lees, Slater y Wyly, 2008; Casgrain y Janoschka, 2013.

59 Janoschka, Sequera y García, 2014. 
una forma de buscar un uso "a la vez analítico y político" de la idea de gentrificación ${ }^{60}$.

Efectivamente, las connotaciones semánticas del término desplazamiento aparecen en sí mismas como objetos de disputa. El desplazamiento es entendido como expulsión, como reemplazo o como recambio, sin cuestionar las implicancias de clase de estos movimientos. A ello se agregan los desafíos metodológicos que conlleva corroborar y medir el desplazamiento en sí. Las dificultades involucran la inexistencia de datos e indicadores relativos al desplazamiento ${ }^{61}$, así como a la componente temporal del proceso, que no permite "capturas" sincrónicas ${ }^{62}$.

Al polemizar con quienes utilizan esta dificultad metodológica para desestimar el desplazamiento por gentrificación, Slater retoma los cuatro tipos de desplazamiento reconocidos por Marcuse: desplazamiento directo del último residente, desplazamiento en cadena, desplazamiento excluyente y presión de desplazamiento. El primero y el segundo dan cuenta de los movimientos producidos a través de mecanismos físicos y económicos, en un momento determinado o en una secuencia temporal. El tercer tipo remite a las dificultades para acceder a las zonas gentrificadas o abandonadas -y el consecuente constreñimiento en las

60 Slater, 2009, p. 295.

61 Slater, 2009.

62 Marcuse, 1985. decisiones- que se plantean para grupos sociales que anteriormente contaban con esas zonas como alternativas residenciales. Finalmente, el cuarto tipo refiere a una presión que no es directa ni siempre económica, que se deriva de una multiplicidad de cambios en el barrio, en el paisaje, en los comercios y servicios, y que conlleva un desfasaje social e identitario entre los antiguos habitantes y su barrio ${ }^{63}$.

Como indica Delgadillo, sería incorrecto esperar del planteo de Marcuse alcances temporales y geográficos mayores al que el propio autor quiso dar, ya que "no podía conceptualizar procesos [como los latinoamericanos] que tal vez estaban ausentes en la realidad neoyorquina" ${ }^{4}$. De todas maneras, la propuesta de Marcuse reviste interés analítico y político general desde al menos tres puntos. En primer lugar, porque facilita recuperar un enfoque diacrónico y continuo, donde los desplazamientos de los sectores más vulnerables no necesariamente se dan por la llegada de grupos de altos ingresos, sino también por grupos intermedios. En esta línea, por ejemplo Insulza revela que los sujetos gentrificadores de Bellavista en Santiago de Chile no son las clases altas, sino los asalariados intermedios, técnicos, profesionales o creativos, a los que denomina trabajadores "de cuello celeste"65.

\footnotetext{
63 Slater, 2009, p. 303.

64 Delgadillo, 2015, p. 12.

65 Inzulza, 2012.
} 
En segundo lugar, Marcuse plantea que el constreñimiento de las opciones de los sectores populares puede darse tanto por el incremento de los precios como por la no puesta en el mercado de las unidades. Por extensión de esta idea, puede pensarse que el desplazamiento no sólo involucra a los sectores populares que directamente pierden residencias, sino también a otros sectores de similar condición social que previo a los procesos de gentrificación contaban con esos espacios como opción residencial de recambio potencialmente accesible. Este punto resulta central en la discusión política, ya que devuelve al problema su carácter de clase (y no de vecindario o grupo espacial particular), y permite discutir por qué es pertinente hablar de desplazamiento y gentrificación en casos de transformación de áreas vacantes de la ciudad ${ }^{66}$, típicamente conceptualizadas dentro de la new-build gentrification ${ }^{67}$.

En tercer lugar, la presión de desplazamiento planteada por Marcuse permite repensar un conjunto de mecanismos (directos-indirectos, intencionalesinvoluntarios) por los cuales aún los hogares vulnerables que pueden no verse directamente desplazados, sufren una serie de agresiones hacia los componentes de su entorno cotidiano, estimulando así

66 Lees, Slater y Wyly, 2008; Slater, 2009.

67 Davidson y Lees, 2005. un desplazamiento "auto-infringido". Janoschka y Sequera afirman que se debe conceptualizar el desplazamiento como "una presión simbólica, forzada por una amplia gama de políticas urbanas, discursos y prácticas", que entre otras cosas "induce a la creciente invisibilidad de algunas prácticas sociales y culturales, la criminalización de otras, la limitación en el uso y la exclusión del espacio público, así como (...) la hegemonía de una civilidad neoliberal de las clases medias urbanas"

Otros trabajos enfatizan que el desplazamiento es una noción socio-territorial, que depende tanto del lugar de expulsión como del de acogida ${ }^{69}$. Lees alerta sobre la articulación territorial de desplazamientos de áreas centrales y re-emplazamientos en áreas periféricas, alejadas de los lugares de trabajo de los desplazados, de sus posibilidades educativas, sus redes sociales y los mejores equipamientos de salud ${ }^{70}$. López-Morales se pregunta si acaso "las políticas de vivienda [no son] formas de utilizar la formalización de los residentes originales para generar su desplazamiento"71, algo que ha sido objeto de extenso debate en el problema de los "con techo" de Chile $e^{72}$.
68 Janoschka y Sequera, 2014, p. 11.
69 Blanco, Apaolaza, Bosoer y González, 2015.
70 Lees, 2012.
71 López Morales, 2015, p. 6.
72 Rodríguez y Sugranyes, 2004. 
En otra línea, la discusión sobre el desplazamiento aparece vinculada a estudios de implementación de políticas públicas dirigidas a introducir "mejoras" en la ciudad sin generar gentrificación. Marcuse fue uno de los pioneros, al proponer algunos lineamientos de "políticas anti-desplazamiento"73. Finalmente, aparece un núcleo de trabajos interesados por la resistencia ciudadana a la gentrificación, así como por las prácticas alternativas para enfrentar el desplazamiento. Dentro de los primeros, aparecen trabajos sobre Chile ${ }^{74}$, México ${ }^{75}$ o España ${ }^{76}$, mientras que dentro de los segundos, aparecen trabajos sobre el movimiento madrileño contra los desahucios ${ }^{77}$, o del clean-up de tugurios en Harare ${ }^{78}$.

En síntesis, en tanto que componente central de los debates sobre la gentrificación, el desplazamiento tiene claras implicancias políticas. En este marco, hay intentos de ajustar la medición del desplazamiento para visibilizar políticamente el proceso y para fortalecer los movimientos de resistencia. Existen estudios de caso que ejemplifican estos desplazamientos y dan cuenta de las pérdidas implicadas en la relocalización.

73 Marcuse, 1984. Véase también Levy, Comey y Padilla, 2006; Bates, 2013.

74 Casgrain y Janoschka, 2013.

75 Nájera, 2014.

76 Janoschka, Sequera y García, 2014; Makhlouf, 2014.

77 Abellán, 2013.

78 Kamete, 2012.
En este sentido, hay vínculos tanto con los estudios de movilidad residencial como con los de movilidad urbana en escala de la cotidianeidad. Hay, asimismo, una paulatina ampliación de la comprensión de los procesos de desplazamiento: a los residenciales se suman los desplazamientos comerciales e industriales y, consecuentemente, de los trabajadores. Incipientemente, hay referencias a desplazamientos en ámbitos rurales, pero en una dinámica relacionada con la urbanización y las prácticas urbanas. No obstante, se abre aquí un punto de contacto con procesos de desplazamiento de población rural que se dan por la expansión de modelos empresariales agro-ganaderos, silvícolas y mineros, de particular relevancia en Latinoamérica.

\section{Discusión: sobre desplazamientos, expulsiones y despojos en los procesos de gentrificación}

La revisión evidencia la existencia de dos sentidos predominantes en el uso del término 
desplazamiento, que coinciden con la raíz etimológica analizada al inicio del trabajo: como cambio de lugar o como pérdida de lugar. Si bien ambos sentidos connotan movimientos en el espacio $-\mathrm{y}$ por ende admiten preguntas comunes acerca de causalidad, motivación o voluntariedad-, interesan fundamentalmente sus mutuas vinculaciones.

Cada sentido tradicionalmente se asocia con ciertos temas más que con otros, pero a la vez se registraron numerosos puntos de contacto. El desplazamiento como movimiento resulta central en los estudios de movilidad, pero cada vez con más frecuencia la expulsión o la ausencia de oportunidades de acceso a la ciudad son entendidas como variables relacionadas. A su vez, dentro de los debates sobre gentrificación, el desplazamiento como pérdida de locus residencial muestra dificultades para abordar otras formas de exclusión menos evidentes, como la laboral, la del espacio público o la del consumo. Buscando una mirada integral, resulta clave considerar la disputa en torno al uso y apropiación de la ciudad en general, y de sus "centros" en particular, como parte de procesos más amplios de acumulación y lucha material y simbólica de clase.

En 1982 al estudiar el fenómeno de la gentrificación Smith sostenía: "por gentrificación me refiero al proceso por el cual los barrios residenciales obreros son rehabilitados por la clase media, propietarios y desarrolladores; hago la distinción teórica entre gentrificación y re-desarrollo [ya que] re-desarrollo no supone la rehabilitación de las viejas estructuras sino la construcción de nuevos edificios"79. Catorce años más tarde, y ante la enorme escala adquirida por el proceso, extendía el término, afirmando que la gentrificación "ya no es una pequeña y atrevida rareza del mercado de la vivienda, sino que se ha convertido en la expresión residencial de un intento mucho más extenso: la reocupación de clase del centro urbano" ${ }^{\text {" }}$.

Este proceso, que Smith terminará por entender como una estrategia global asociada a un nuevo urbanismo revanchista ${ }^{81}$, adquiere connotaciones aún más dramáticas en Latinoamérica, dónde la gentrificación no sólo remite a la pérdida de centralidad sino también al confinamiento en periferias que se caracterizan por sus altísimos niveles de privación material, precariedad urbana y vulnerabilidad ambiental.

De igual manera, las líneas que investigan desplazamiento en torno a cuestiones ambientales, conflictos militares o proyectos de desarrollo dan cuenta de numerosas situaciones en las que la expulsión se produce en el marco del despojo de ciertos grupos sociales, y de apropiación de recursos

\footnotetext{
79 Smith, 1982, p. 139.

80 Smith, 1996, p. 39.

81 Smith, 2002.
} 
materiales e inmateriales por parte de grupos de poder. La idea general de apropiación y uso excluyente del territorio por actores dominantes, clave para la gentrificación, está presente también en los conflictos político-económicos, en las planificaciones estatales o en el avance de estructuras empresariales en ámbitos rurales, denotando un rasgo inherente al capitalismo contemporáneo, que instrumentaliza y sistematiza el desplazamiento como parte del proceso general de acumulación.

Entendido así, el desplazamiento no puede ser conceptualizado sólo como un efecto colateral indeseado de la acumulación capitalista "pura" (acumulación por explotación del trabajo, por captación de rentas extraordinarias, etc.). Por el contrario, el desplazamiento -incluyendo aquel generado por la gentrificación- debe inscribirse dentro de los múltiples procesos de acumulación por desposesión, es decir formas de acumulación que no dependen de una lógica económico-productiva sino de avanzadas de saqueo y depredación de bienes o recursos no producidos por el capitalismo ${ }^{82}$. Esta ubicuidad del desplazamiento en los procesos de desposesión ha llevado a que algunos autores incluso propongan la idea de "acumulación por desplazamiento"83, como una manera de resaltar esta dimensión de la gentrificación.

82 Harvey, 2003.

83 Janoschka y Sequera, 2016.
En este escenario, la gentrificación representa quizá una de las formas de acumulación por desposesión más novedosas y sutiles, ya que el despojo no se realiza mediante la embestida contra conquistas laborales o antiguos bienes comunes, sino mediante la usurpación excluyente de la centralidad, es decir de un producto colectivo de la sociedad urbana.

Con los desplazamientos de personas a escala planetaria como telón de fondo, Sassen observa que en los últimos treinta años "ha habido un fortalecimiento de las dinámicas que expulsan gente de la economía y de la sociedad, y ahora esas dinámicas están programadas como parte del funcionamiento normal de esas esferas", configurando lo que denomina "formaciones predatorias" ${ }^{4}$. En este escenario, el confinamiento de los pobres urbanos en las periferias precarias podría representar el último eslabón, el locus finalis, de una cadena de sucesivos desplazamientos.

\section{Conclusiones}

Como conclusión, y más allá de las anteriores reflexiones de carácter conceptual y global, resulta útil plantear cinco recomendaciones prácticas surgidas de las líneas revisadas, que podrían ayudar a formular nuevas preguntas de la relación entre

\footnotetext{
84 Sassen, 2015, p. 91-92.
} 
gentrificación, desplazamiento y desposesión. A saber:

1. Las consecuencias extra residenciales del desplazamiento, que dan cuenta de los numerosos efectos negativos asociados: desarticulación laboral, destrucción de lazos sociales, estrés y vulnerabilidad psicofísica e identitaria. Se trata de dimensiones que ocupan un lugar importante en algunas de las líneas de investigación revisadas, pero que están menos desarrolladas en relación con la gentrificación. La identificación de esas prácticas o la estimación de las pérdidas y "costos" asociados a la reconstrucción de los sistemas residenciales y de movilidad son aspectos relevantes para incorporar al análisis.

2. La importancia de la trayectoria histórica y el contexto territorial. Es marcada la necesidad de una reconstrucción en perspectiva amplia de los procesos de desplazamiento, para visibilizar desplazamientos de población que de otro modo podían pasar desapercibidos. A su vez, se enfatiza la necesidad de considerar tanto el lugar de expulsión como el de acogida. En este punto, prima el interés por prevenir futuros desplazamientos antes que por describir aquellos ya en curso, así como por evaluar las posibilidades de reversión del proceso.
3. Las condiciones preexistentes y el detonante. Interesa analizar cómo situaciones de fragilidad social, económica y jurídica previa habilitan procesos de desplazamiento. Por otro lado, se encontraron decenas de antecedentes en los que se describe cómo un evento específico puede desencadenar procesos de desplazamiento permanentes: catástrofes naturales, conflictos armados o eventos internacionales pueden funcionar como detonantes o justificativos de posteriores procesos expulsivos vía mercado.

4. La tensión voluntario-involuntario-forzado. Especialmente enfatizada en los procesos político-económicos, la investigación actual da cuenta de la importancia de esta dimensión del desplazamiento pero también de las dificultades de pensar de manera dicotómica una tensión que se construye con muy diversos componentes, sin límites definidos, y con restricciones y posibilidades que acotan notablemente las decisiones que pueden tomar los sujetos y los grupos que sufren desplazamiento. En este punto, como estrategia de resistencia, resulta clave romper el anonimato causal que oculta las responsabilidades político-económicas.

5. La tipificación de la figura del desplazado. Este antecedente ayuda a pensar en la figura 
de otros desplazados, en la construcción de sus perfiles, en el reconocimiento de sujetos y grupos en esta situación y en su visibilización, base para la resistencia y la disputa política. De todas maneras, la emergencia de la figura del desplazado por gentrificación no depende tanto de aportes académicos exógenos como de la consolidación de un colectivo social de afectados con identidad propia y capacidad de autogestión.

Como cierre, se destaca que la discusión a partir de la diversidad de usos del concepto de desplazamiento identificados en el artículo podría ser de utilidad para cualquiera de los siete campos de interés analizados. De manera específica, y de acuerdo con el propósito del trabajo, el debate sobre la gentrificación podría verse enriquecido al repensar y articular esta amplia variedad de sentidos. Se trata de sentidos que abren nuevas preguntas sobre los desplazamientos y los desplazados, y que, al inscribirse en las discusiones más amplias sobre desposesión y disputa urbana de clases, cobran una significación eminentemente política y práctica.

\section{Bibliografía}

ABELLÁN, Jacobo. Ciudad, crisis y desobediencia: una aproximación a las luchas por la vivienda en Madrid. En: HIDALGO, Rodrigo, ed. y JANOSCHKA,

ARTíCULO: Políticas y geografías del desplazamiento. Contextos y usos conceptuales para el debate sobre gentrificación/Jorge Blanco, Ricardo Apaolaza
Michael, ed. La ciudad neoliberal. Gentrificación y exclusión en Santiago de Chile, Buenos Aires, Ciudad de México y Madrid. Santiago, Pontificia Universidad Católica de Chile. 2013. GEOlibros.

ABELLÁN, Antonio y ROJO, Fermina. Migración y movilidad residencial de las personas de edad en Madrid. Anales de Geografía de la Universidad Complutense. (17): 175-193, 1997.

ASCHER, François. Les sens du mouvement. Paris, Belin. 2004.

BARUA, Prabal y SHAHJAHAN, Mohammad. Superior settlement for climate displaced people of Bangladesh. [En línea]. Social Sciences Research Network. Diciembre, 2013. Disponible en: http://papers. ssrn.com/sol3/papers.cfm?abstract_id=2370767.

BATES, Lisa. Gentrification and displacement study. Portland, BPS. 2013

BLACK, Richard; ARNELL, Nigel; ADGER, Neil; THOMAS, David y GEDDES, Andrew. Migration, immobility and displacement outcomes following extreme events. Environmental Science E Policy. 27(1): S32-S43, 2013. DOI 10.1016/j. envsci.2012.09.001.

BLANCO, Jorge; BOSOER, Luciana y APAOLAZA, Ricardo. Movilidad, apropiación y uso del territorio: una aproximación a partir del caso de Buenos Aires. Scripta Nova Revista electrónica de geografía y ciencias sociales. 18(493), 2014. ISSN 1138-9788.

BLANC0, Jorge; APAOLAZA, Ricardo; BOSOER, Luciana y GONZÁLEZ, Ariel. Movilidades, desplazamientos y territorios. Algunos aportes para el 
debate sobre la gentrificación. [En línea] Working Paper Series Contested Cities. (15004), 2015. ISSN 2341-2755. Disponible en: http://contested-cities. net/working-papers/wp-content/uploads/sites/8/2015/01/WPCC-15004-BlancoApaolazaBosoerGonzalez_MovilidadesDesplazamientosTerritorios.pdf

BRACHET, Julien. Géographie du mouvement, géographie en mouvement. Francia, Arras. 2008.

BRITO, Fausto. O deslocamento da população brasileira para as metrópoles. Estudos Avançados. 20(57). 221-236, 2006. DOI 10.1590/ S0103-40142006000200017.

BRONEN, Robin. Forced migration of alaskan indigenous communities due to climate change. En: AFIFI, Tamer, ed. y JÄGER, Jill, ed. Environment, Forced Migration and Social Vulnerability. London, Springer. 2010. ISBN 978-3-642-12416-7.

BRUN, Cathrine. Reterritorializing the relationship between people and place in refugee studies. Geografiska Annaler: Series B, Human Geography. 83(1): 1525,2001. DOI 10.1111/j.0435-3684.2001.00087.x.

CASGRAIN, Antoine y JANOSCHKA, Michael. Gentrificación y resistencia en las ciudades latinoamericanas. El ejemplo de Santiago de Chile. Andamios. 10(22): 19-44, 2013.

CERNEA, Michael. The risk and reconstruction model for ressetling displaced populations. World Development. 25(10): 1569-1587, 1997. DOI 10.1016/ S0305-750X(97)00054-5.
DAVIDSON, Mark y LEES, Loreta. New-build gentrification and London's riverside renaissance. EnvironmentEPlanning A. 37(7): 1165-1190, 2005. DOI 10.1068/a3739.

DELGADILL0, Víctor. Desafíos para el estudio de desplazamientos sociales en los procesos de gentrificación. En: Realidades de procesos de desplazamiento en América Latina. Madrid, Contested_ Cities. 2015

DI VIRGILIO, Mercedes. Diferencias sociales en los procesos de movilidad residencial intraurbana en el AMBA. Quivera. 16(1): 11-37, 2014.

DUREAU, Françoise. Les systèmes résidentiels: concepts et applications. En: LÉVY, Jacques; DUREAU, Françoise. Laccès à la ville: les mobilités spatiales en questions. Paris, L'Harmattan. 2002. ISBN 2-7475-2806-5.

ECHAVARRíA, Juan. Contribución al análisis de las migraciones. Lecturas de Economía. (2). 119-140, 1980.

EGEA, Carmen; SOLEDAD, Javier. Migraciones y conflictos: El desplazamiento interno en Colombia. Convergencia. 15(47): 207-235, 2008.

FENNER SÁNCHEZ, Gabriela. La reubicación de poblaciones como estrategia de ordenamiento territorial. [En línea]. Revista Geográfica de América Central. 1-18, 2011. Disponible en: http://www. revistas.una.ac.cr/index.php/geografica/article/ view/2700.

FLORES, Jorge. Infraestructura carretera: construcción, financiamiento y resistencia en México y 
América Latina. Transporte y Territorio. (13): 122148, 2015. ISSN 1852-7175.

GÓMEZ, Anahí; WAGNER, Lucrecia; TORRES, Beatriz; MARTIN, Facundo y ROJAS, Facundo. Resistencias sociales en contra de los megaproyectos hídricos en América Latina. ERLACS. (97): 75-96, 2014. DOI http://doi.org/10.18352/erlacs.9797.

GUTIÉRREZ, Andrea. ¿Qué es la movilidad? Elementos para (re)construir las definiciones básicas del campo del transporte. Bitácora Urbano Territorial. 2(21): 61-74, 2012.

HARVEY, David. The new imperialism. Oxford, Oxford University Press. 2003.

HYNDMAN, Jennifer. The securitization of fear in posttsunami Sri Lanka. Annals of the Association of American Geographers. 97(2): 361-372, 2007. DOI 10.1111/j.1467-8306.2007.00542.x.

INZULZA, Jorge. «Latino-gentrification»?: Focusing on physical and socioeconomic patterns of change in Latin American inner cities". Urban Studies. 49(10): 2085-2107, 2012. DOI 10.1177/0042098011423425.

INZULZA, Jorge y LÓPEZ, Néstor. Gentrificación de escala intermedia global en Latinoamérica. El caso de la reconstrucción de Managua. Revista de Urbanismo. (31), 2014.

JANOSCHKA, Michael y SEQUERA, Jorge. Gentrification in Latin America: adressing the politics and geographies of displacement. Urban Geography. 2016. DOI 10.1080/02723638.2015.1103995.
--- Procesos de gentrificación y desplazamiento en América Latina. Una perspectiva comparativista. En: MICHELINI, Juan, coord. Desafíos metropolitanos. Un diálogo entre Europa y América Latina. Madrid, Catarata. 2014.

JANOSCHKA, Michael; SEQUERA, Jorge y GARCÍA, Eva. Gentrificación, resistencias y desplazamiento en España. Propuestas analíticas. En: Gentrificación, resistencias y desplazamiento en España. Madrid, Contested_Cities. 2014.

JOHNSTON, Caleb. Politics in the informalizing metropolis: Displacement, resettlement and unstable negotiations in uncivil Ahmedabad. International Journal of Urban and Regional Research. 38(2): 539557, 2014. DOI 10.1111/1468-2427.12010.

KAMETE, Amin. Not exactly like the Phoenix, but rising all the same: reconstructing displaced livelihoods in post-cleanup Harare. Environment \& Planning D. 30(2): 243-261, 2012. DOI 10.1068/ d2408.

KOLMANNSKOG, Vikram y TREBBI, Lisetta. Cambio climático, desastres naturales y desplazamiento". International Review of the Red Cross. (879): 307327,2010

LEES, Loretta. The geography of gentrification: Thinking through comparative urbanism. Progress in Human Geography. 36(2). 155-171, 2012.

LEES, Loretta; SLATER, Tom y WYLY, Elvin. Gentrification. New York, Taylor \& Francis. 2008. 344 p. ISBN 978-0-415-95036-7. 
LEVY, Jacques. Les nouveaux espaces de la mobilité. En: BONNET, Michel, dir. y DESJEUX; Dominique, dir. Les territoires de la mobilité. París, Presses Universitaires de France. 2000.

LEVY, Diane; COMEY, Jennifer y PADILLA, Sandra. In the face of gentrification: case studies of local efforts to mitigate displacement. Washington, The Urban Institute. 2006.

LÓPEZ MORALES, Ernesto. Gentrificación y desplazamiento en América Latina: tres factores causales concatenados. En: Realidades de procesos de desplazamiento en América Latina. Madrid, Contested_Cities. 2015.

MAKHLOUF, Muna. Transformaciones urbanas y procesos de gentrificación desde la resistencia. Aproximaciones a un movimiento vecinal en la Barceloneta, Barcelona. En: Gentrificación, resistencias y desplazamiento en España. Madrid, Contested_ Cities. 2014.

MARCUSE, Peter. Gentrification, abandonment and displacement. Connection, cause and policy responses in New York City. [En línea]. Journal of urban and contemporary law. 28: 195-240, enero 1985. Disponible en: http://openscholarship. wustl.edu/law_urbanlaw/vol28/issl/4.

--- To control gentrification: anti-displacement zoning and planning for stable residential districts. Review of Law and Social Change. 13(4): 931-945, 1984.

MÓDENES, Juan. Movilidad espacial, habitantes y lugares: retos conceptuales y metodológicos para la geodemografía. Estudios Geográficos. 69(264): 157178, 2008. DOI 10.3989/egeogr.2008.i264.83.

MUGGAH, Robert. A tale of two solitudes: Comparing conflict and development-induced internal displacement and involuntary resettlement. International Migration. 41(5): 5-31, 2003. DOI 10.1111/j.0020-7985.2003.00259.x.

MYERS, Norman. Environmental Refugees. Population and Environment. 19(2). 167-82, 1997. DOI 10.1023/A:1024623431924.

NÁJERA, Martín. Impulso a la competitividad mundial de la ciudad de México. Una gentrificación liderada por el Estado frente a la resistencia ciudadana". En: Perspectivas del Estudio de Gentrificación en México y Latinoamérica. Madrid, Contested_Cities. 2014.

OLIVER-SMITH, Anthony. Debating environmental migration: society, nature and population displacement in climate change. Journal of International Development. 24(8): 1058-1070, 2012. DOI 10.1002/jid.2887.

OSORIO, Flor. Actores y elementos en la construcción de una nueva categoría social en Colombia: los desplazados. Scripta Nova. 94(1). 2001.

PENZ, Peter. Development, displacement, coercion and harm: a theoretical treatment. En: International Association for the Study of Forced Migration ( $8^{\mathrm{a}}$, Chiang Mai, Thailand, 2003)

RODRÍGUEZ, Alfredo y SUGRANYES, Ana. El problema de vivienda de los «con techo». Eure. 30(91): 53-65, 2004. DOI 10.4067/S0250-71612004009100004. 
RUIZ, Nubia. La migración forzada en Colombia a causa del desplazamiento forzado. En: III Congreso de la Asociación Latinoamericana de Población. Córdoba. 2008.

RUWANPURA, Kanchana. Putting houses in place: rebuilding communities in post-tsunami Sri Lanka. Disasters. 33(3). 436-456, 2009. DOI 10.1111/j.1467-7717.2008.01082.x.

SASSEN, Saskia. Expulsiones. Brutalidad y complejidad en la economía global. Buenos Aires, Katz. 2015.

SCUDDER, Thayer y COLSON, Elizabeth. From welfare to development: a conceptual framework for the analysis of dislocated people. En: HANSEN, Art y OLIVER-SMITH, Anthony. Involuntary migration and ressetlement. Colorado, Westview Press. 1982.

SILVEY, Rachel. Power, difference and mobility: feminist advances in migration studies. Progress in Human Geography. 28(4): 1-17, 2004. DOI 10.1191/0309132504ph490oa.

SLATER, Tom. Missing Marcuse: On gentrification and displacement. City. Analysis of Urban Trends Culture Theory Policy and Action. 13(2): 294-311, 2009. DOI 10.1080/13604810902982250.

SMITH Neil. New globalism, new urbanism: gentrification as global urban strategy. Antipode. 34(3): 427445, 2002. DOI DOI: 10.1111/1467-8330.00249.

--- The new urban frontier: Gentrification and the revanchist City. London, Routledge. 1996.
--- Gentrification and uneven development. Economic Geography. 58(2): 139-155, 1982. DOI 10.2307/143793.

SUÁREZ, Manuel; MURATA, Masanori y DELGADO CAMPOS, Javier. Why do the poor travel less? Urban structure, commuting and economic informality in Mexico City. Urban Studies. 53(12): 25482566, 2016. DOI 10.1177/0042098015596925.

TERMINSKI, Bogumil. Development-induced displacement and ressetlement: theoretical frameworks and current challenges. Geneva, University of Geneva. 2013.

THOMAS, Marie-Paule; PATTARONI, Luca y KAUFMANN, Vincent. Modes de vie, mobilité et organisation quotidienne des familles. En: GERBER, Philippe y CARPENTIER, Samuel. Les interactions entre mobilités quotidienne et résidentielle. Rennes, Presses Universitaires de Rennes. 2011.

TIMMS, Benjamin. The (mis)use of disaster as opportunity: Coerced relocation from Celaque National Park, Honduras. Antipode. 43(4): 1357-1379, 2011. DOI 10.1111/j.1467-8330.2011.00865.x.

VAINER, Carlos. Deslocamentos compulsórios, restrições á libre circulação. En: XI Encontro Nacional de Estudos Populacionais da ABEP. 1998.

VELEDA, Susana. Desplazamiento y relación con los lugares: un estudio cualitativo. Scripta Nova. 94(102), 2001

VESCHAMBRES, Vincent. La notion d'appropriation. [En línea] Norois. (195), 2005. Disponible en: https://norois.revues.org/589. 
VICTORIA, María y MOLINA, Carlos. Reasentamiento involuntario: integración y civilización. Bitácora Urbano Territorial. 1(7): 19-25, 2003.

ZALIK, Anna. Zones of exclusion: offshore extraction, the contestation of space and physical displacement in the Nigerian Delta and the Mexican Gulf. Antipode. 41(3): 557-582, 2009. DOI 10.1111/j.1467-8330.2009.00687.x. 